\title{
Stability analysis on the radioactive iodine-labelled prostate cancer-specific recombinant oncolytic adenovirus
}

\author{
JIAHE ZHOU ${ }^{1,2^{*}}$, LIN HAO $^{1,2^{*}}$, ZHENDUO SHI $^{1,2^{*}}$, SONGYI NING $^{4 *}$, HOUGUANG HE $^{1}$, YAN ZHAO $^{3}$, \\ YANG DONG ${ }^{1}$, ZHIGANG LI $^{1}$, JIUXIANG HE ${ }^{5}$, GUANGHUI ZANG ${ }^{1}$ and CONGHUI HAN ${ }^{1-3}$ \\ ${ }^{1}$ Department of Urinary Surgery, Xuzhou School of Clinical Medicine Affiliated to Xuzhou Medicine University; \\ ${ }^{2}$ Department of Urinary Surgery, Xuzhou Central Hospital, Xuzhou, Jiangsu 221009; ${ }^{3}$ Kunshan Ruike Research \\ and Development of Medicine Co., Ltd., Kunshan, Jiangsu 215300; ${ }^{4}$ Jiangsu University School of Medicine, \\ Zhenjiang, Jiangsu 212013; ${ }^{5}$ Chongqing Western Biomedical Technology Co., Chongqing 400010, P.R. China
}

Received August 12, 2016; Accepted March 24, 2017

DOI: $10.3892 / \mathrm{ol} .2017 .6998$

\begin{abstract}
The aim of the present study was to construct the ${ }^{125}$ I-replication-selective oncolytic adenovirus (RSOAds)human telomerase reverse transcriptase (hTERT)/prostate specific antigen (PSA) nuclide-oncolytic virus marker by labelling the hTERT/PSA double-regulation replicative oncolytic adenovirus with ${ }^{125} \mathrm{I}$ nuclide, and investigate the influence of viral markers under various reaction conditions on labelling efficiency. N-bromosuccinimide (NBS) was used as the oxidizer for ${ }^{125}$ I labelling, and the best conditions for labelling were identified through the reactions between oncolytic adenovirus at various concentrations and NBS. Dosage of ${ }^{125} \mathrm{I}$, reaction duration, $\mathrm{pH}$ values and reaction volume were respectively evaluated to determine their effects on the labelling efficiency of ${ }^{125} \mathrm{I}-\mathrm{RSOAds}$-hTERT/PSA nuclide-oncolytic adenovirus markers. Purified nuclide-oncolytic adenovirus markers were isolated by gel-filtration chromatography; paper chromatography was performed to assay the radiochemical purity of ${ }^{125}$ I-RSOAds-hTERT/PSA markers at various time points. Radiochemical purity of ${ }^{125} \mathrm{I}$-RSOAds-hTERT/PSA was $>95 \%$, and could be maintained at $4{ }^{\circ} \mathrm{C}$ for 7 days. The best reaction conditions were set as follows: $0.5 \mu \mathrm{l}$ of ${ }^{125} \mathrm{I}$ $(\sim 0.2 \mathrm{~m} \mathrm{Ci}, 7.4 \mathrm{MBq}) ; 25 \mathrm{qg}$ of NBS; $100 \mu \mathrm{l}$ of $8 \times 10^{9} \mathrm{VP} / \mathrm{ml}$ ${ }^{125} \mathrm{I}$-RSOAds-hTERT/PSA virus solution; $30 \mathrm{~min}$ of reaction duration; $\mathrm{pH} 7.5 ; 120 \mu \mathrm{l}$ of PBS. Labelling hTERT/PSA double-regulation replicative oncolytic adenovirus with ${ }^{125} \mathrm{I}$
\end{abstract}

Correspondence to: Dr Conghui Han, Department of Urinary Surgery, Xuzhou School of Clinical Medicine Affiliated to Xuzhou Medicine University, 199 South Jiefang Road, Xuzhou, Jiangsu 221009, P.R. China

E-mail: hanchdoctor@qq.com

*Contributed equally

Key words: castrate-resistant prostate cancer, ${ }^{125} \mathrm{I}$, replicationselective oncolytic adenovirus, prostate specific antigen, human telomerase reverse transcriptase was identified to be available, and the radiochemical purity of acquired virus markers could be maintained under specific conditions.

\section{Introduction}

As one of the most common types of cancer of the male reproductive system, the incidence of prostate cancer is ranked second of all malignant tumors of males. At present, the incidence of prostate cancer, in the USA, has surpassed lung cancer as the highest incidence, threatening the health of males $(1,2)$ According to the latest data issued by The National Cancer Center of China, prostate cancer, since 2008, is ranked first in terms of the incidence of tumors in urinary system (3). Conventional treatment for prostate cancer includes hormonotherapy, radiotherapy and chemotherapy as well as surgery. Nevertheless, there remain great difficulties in treatment of castrate-resistant prostate cancer (CRPC) due to its high post-surgery recurrence rate or no response to the endocrine therapies, leaving few treatment options. Radiotherapy, as one of the major therapies for prostate cancer, has been widely applied in treatment of prostate cancer in all stages, in which the radiosensitivity is a key factor intimately affecting the efficacy of radiotherapy. Thus, how to increase the radiosensitivity of tumors is a study hotspot in the domain of tumor therapeutics. In recent years, as the basic study of tumors has acquired continuous development, especially the development in molecular biology, how to improve the efficacy of radiotherapy and chemotherapy by application of targeted therapy with gene drugs has become a new development trend (4). Replication-selective oncolytic adenovirus (RSOAds) represents a method of targeted gene therapy of tumors, while the construction of RSOAds dual-targeted and dual-regulated prostate specific antigen (PSA) and human telomerase reverse transcriptase (hTERT) could further enhance the targeting effect of therapeutic gene to the prostatic tumor tissues, which is expected to generate better efficacy in clinical treatment (5). In the present study, the aim was set to construct the ${ }^{125}$ I-RSOAds-hTERT/PSA nuclide-oncolytic virus marker by labelling the hTERT/PSA double-regulation replicative oncolytic adenovirus with ${ }^{125} \mathrm{I}$, results of which would serve 
as experiment basis for later studies of therapies for CRPC by ${ }^{125}$ I-RSOAds-hTERT/PSA markers to explore new, efficient and safe methods for treatment of CRPC.

\section{Materials and methods}

\section{Agent and instruments}

Major agents. PBS (Zhongshan Goldenbridge, Beijing, China), N-bromosuccinimide (NBS) (Sigma, St. Louis, MO, USA), Na $\cdot 4^{125}$ I (Chengdu Zhonghe Gaotong Isotope Co., Ltd., Chengdu, China), acetone (ChengDu KeLong Chemical Co., Ltd., Chengdu, China), human serum albumin (HSA; Hualan Biological Engineering, Inc., Henan, China), SephadexG-10 gel (Nanjing SenBeiJia Biological Technology Co., Ltd., Nanjing, China).

Instruments. $\gamma$ immunity meter (PerkinElmer, Inc., Waltham, MA, USA), whirlpool-like mixers (Qiliner Instruments of Haimen Co., Ltd., Haimen, China), automatic sampling instrument (Shanghai Qingpu Luxi Instrument Plant, Shanghai, China), and chromatography column $(1 \times 40 \mathrm{~cm})$ (Shanghai Wuxiang Chemical Co., Ltd., Shanghai, China).

Identification of the best conditions for labelling. Effect of dosage of ${ }^{125}$ I on the labelling rate was carried out. Briefly, $100 \mu \mathrm{l}$ of $8 \times 10^{8} \mathrm{VP} / \mathrm{ml}{ }^{125}$ I-RSOAds-hTERT/PSA virus solution and $0.1 \mu \mathrm{l}$ of $\mathrm{Na}^{125} \mathrm{I}(\sim 0.04 \mathrm{~m} \mathrm{Ci})$ were added in $1.5 \mathrm{ml}$ centrifuge tube and mixed uniformly. Then $100 \mu \mathrm{l}$ of NBS solution at the concentration of $0.5 \mathrm{mg} / \mathrm{ml}$ and $20 \mu \mathrm{l}$ of PBS $(0.05 \mathrm{~m}$, $\mathrm{pH}=7.6$ ) were added and the tube was sealed and placed into whirlpool-like mixer for uniform mixing, after $3 \mathrm{~min}$ of reaction, $10 \mu \mathrm{l}$ of $2 \%$ HAS was added to stop the reaction, the dosages of NBS solution and ${ }^{125}$ I-RSOAds-hTERT/PSA virus solution as well as other conditions were kept unchanged, and we assayed the influence of dosage of ${ }^{125}$ I (setting dosages: $0.1,0.5,1,2,3,4$ and $5 \mu \mathrm{l}$ ) on the labelling rate of ${ }^{125}$ I-RSOAds-hTERT/PSA nuclide-oncolytic adenovirus markers with the same methods and measurements of the labelling rate by paper chromatography: Paper chromatography conditions were set as follows: filter paper (size, 3; Xinhua Co., Hangzhou, China) as stationary phase; acetone as developers; normal saline 1:1 (v/v).

A marker was made by a pencil every $1 \mathrm{~cm}$ on the filter paper with a total length of $15 \mathrm{~cm}$. The above labelled reaction solution $(0.2 \mu \mathrm{l})$ was added at the start point of filter paper, then spread the paper in the developers $(\sim 300 \mu 1)$. The fully-spread filter paper was taken out and dried until the front edge of solvent moved $2 \mathrm{~cm}$ from the edge of paper. Then, the paper was cut into pieces by the markers, and the counting of radioactivity (cpm) of each piece was measured by $\gamma$-counter. The following formula was used for labelling rate calculation:

\section{Labelling rate $(\%)$ \\ 125I-RSOAds-hTERT/PSA peak counting (cpm)

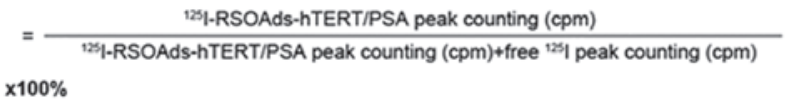

Influences of NBS dosage on the labelling rate. According to the methods described above, influences of various dosages of NBS (setting dosage: 30, 50, 70 and $100 \mu \mathrm{l}$ ) on the labelling rate of ${ }^{125}$ I-RSOAds-hTERT/PSA nuclide-oncolytic adenovirus markers were respectively assayed. Measurements of the labelling rate where performed by paper chromatography.

Influence of virus solution volume on the labelling rate. According to the methods previously described, influence of various volumes of ${ }^{125}$ I-RSOAds-hTERT/PSA virus solution (setting volume: $25,50,75,100,130$ and $150 \mu 1$ ) on the labelling rate of ${ }^{125}$ I-RSOAds-hTERT/PSA nuclide-oncolytic adenovirus markers were respectively assayed. Measurements of the labelling rate were performed by paper chromatography.

Influence of other reaction conditions on the labelling rate. According to the above described methods, the influence of reaction duration (setting value: $1,2,3,4,5,6,7$ and $8 \mathrm{~min}$ ), $\mathrm{pH}$ values (setting value: 6.5, 7.0, 7.5, 8.0 and 8.5 ) and PBS reaction volumes (setting value: $20,30,40,50,60,80,100$, 150 and $200 \mu \mathrm{l}$, the effect of PBS was to adjust the $\mathrm{pH}$ value) on the labelling rate of ${ }^{125}$ I-RSOAds-hTERT/PSA nuclide-oncolytic adenovirus markers were respectively assayed.

Isolation and purification of makers. Gel-filtration chromatography was applied in isolation and purification of ${ }^{125}$ I-RSOAds-hTERT/PSA. After labelling reactions, gel-filtration chromatography was performed with SephadexG-10 chromatography columns $(1 \times 40 \mathrm{~cm})$ in the mixture of the above reactions. A certain volume of HSA was added in the columns for saturation to inhibit the non-specific absorption of RSOAds-hTERT/PSA to the columns before loading the sample. Then the sample was loaded and diluted with PBS $(\mathrm{pH}=7.6)$ to $0.5 \mathrm{ml}$. Thereafter, eluent was collected continuously into 50 tubes by automatic sampling instruments, each tube containing $1 \mathrm{ml}$ of eluent ( $\sim 15$ drops). $10 \mu \mathrm{l}$ of eluent was extracted from each tube to measure the radioactive counts $\left(\mathrm{min}^{-1}\right)$ and to identify the peak positions of markers and free ${ }^{125} \mathrm{I}$. The sample with the first peak (the ${ }^{125}$ I-RSOAds-hTERT/PSA) was selected to be preserved at $4^{\circ} \mathrm{C}$ for use after filtration and sterilization by microporous membrane $(0.22 \mu \mathrm{m})$.

Measurements of radiochemical purity. The markers acquired from the experiment 2 were preserved at $4^{\circ} \mathrm{C}$, and radiochemical purity was determined on days 1, 2, 3, 4, 5, 6 and 7 after labelling by paper chromatography. Radiochemical purity of the sample with the first peak of radioactivity was assayed by paper chromatography, and the method was the same as the assay of labelling rate.

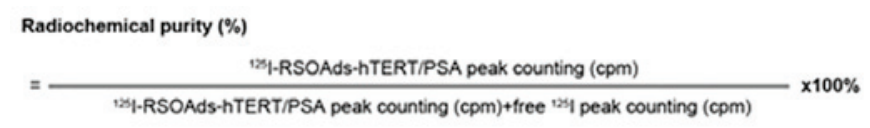

\section{Results}

Identification of the best conditions for labelling. Influence of ${ }^{125}$ I dosage on the labelling rate. Labelling rate of ${ }^{125}$ I-RSOAds-hTERT/PSA was assayed by paper chromatography according to the differences in diffusion coefficients between ${ }^{125}$ I-RSOAds-hTERT/PSA markers and free ${ }^{125} I$, i.e., the latter larger than the former and two peaks appeared on 


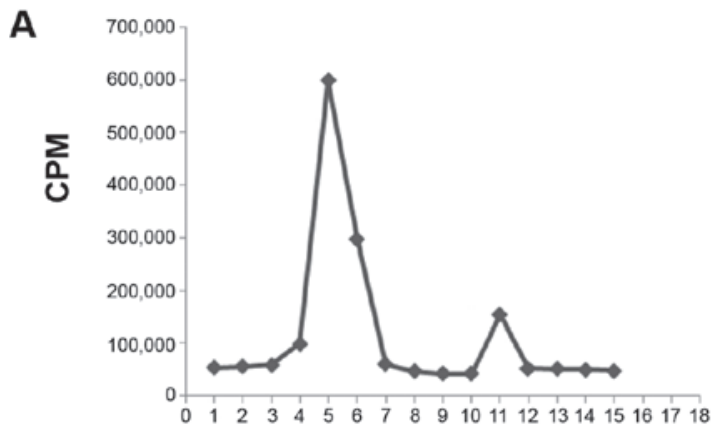

No. of filtration paper

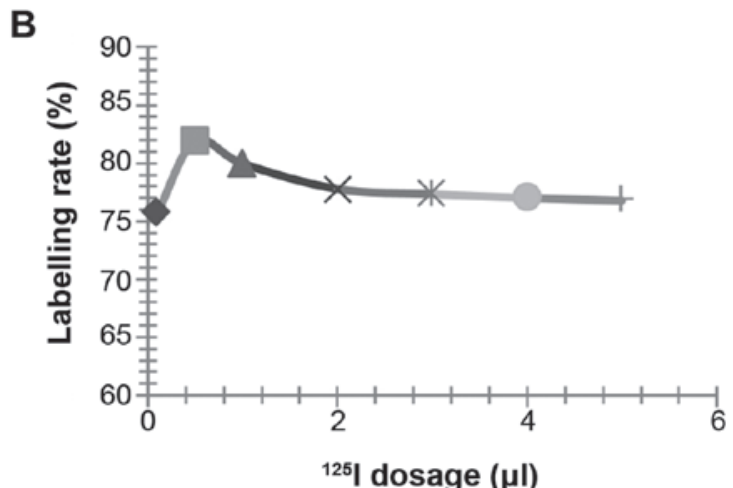

${ }^{125} \mathrm{I}$ dosage $(\mu \mathrm{l})$

Figure 1. Influence of ${ }^{125} \mathrm{I}$ dosage on the labelling rate.

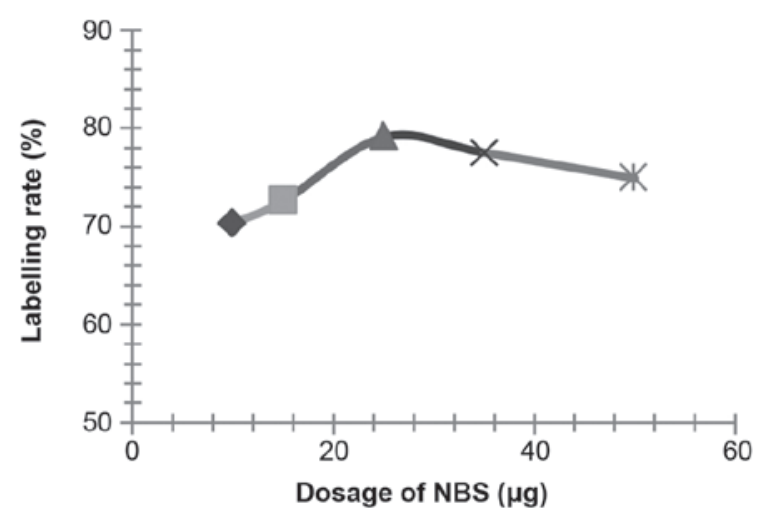

Figure 2. Influence of NBS dosage on the labelling rate.

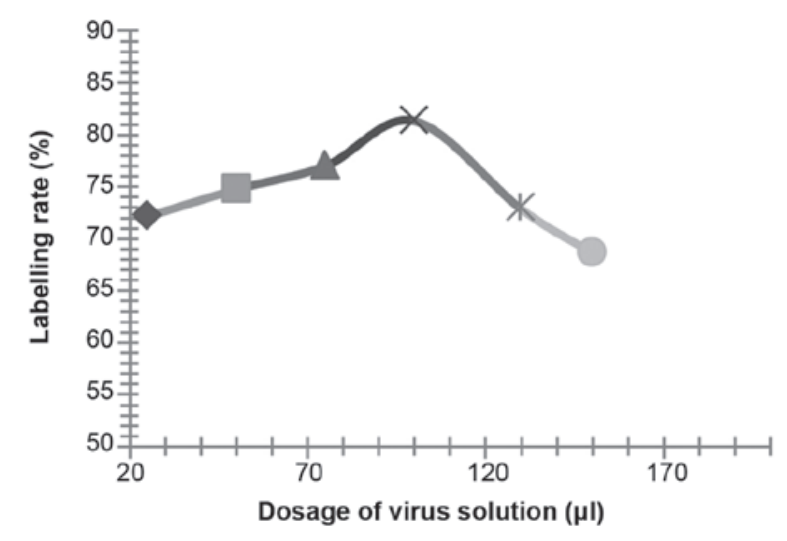

Figure 3. Influence of dosage of virus solutions on the labelling rate.

the paper, peak I and peak II: Peak I represents the peak of ${ }^{125}$ I-RSOAds-hTERT/PSA and peak II represents the peak of free ${ }^{125} \mathrm{I}$.

It was identified (Fig. 1) that the labelling rate was relatively high when the dosage of ${ }^{125} \mathrm{I}$ was $0.5 \mu 1(\sim 0.2 \mathrm{~m} \mathrm{Ci}$, 7.4 MBq).

Influence of other factors on the labelling rate. The influence of NBS, dosage of virus solution, reaction duration, $\mathrm{pH}$ and PBS volume on the labelling rate is shown in Figs. 2-6.

According to the results of the above experiments, the best conditions were identified as: $0.5 \mu 1$ of ${ }^{125} \mathrm{I}(\sim 0.2 \mathrm{~m} \mathrm{Ci}, 7.4 \mathrm{MBq})$,

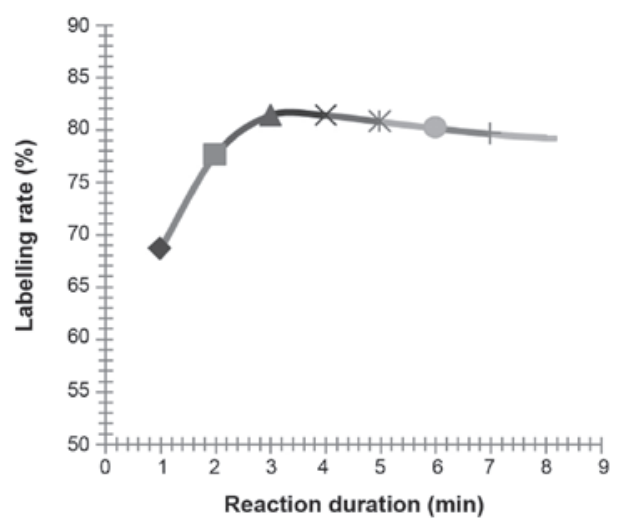

Figure 4. Influence of reaction duration on the labelling rate.

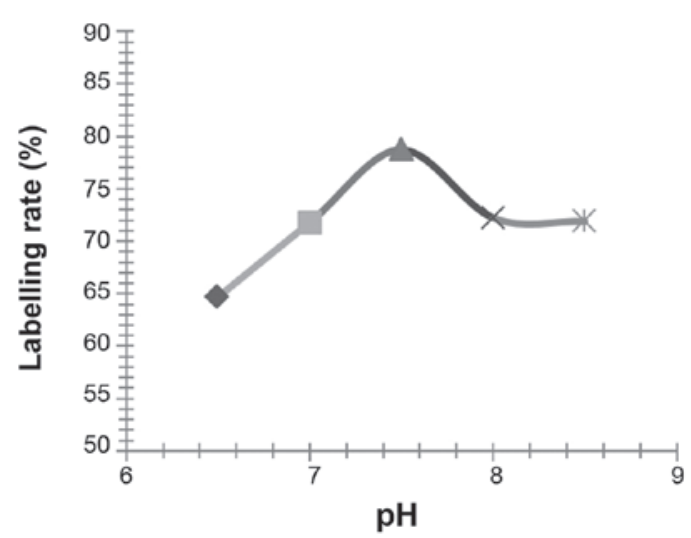

Figure 5. Influence of $\mathrm{pH}$ on the labelling rate.
$25 \mu \mathrm{g}$ of NBS, $100 \mu \mathrm{l}$ of $8 \times 10^{9} \mathrm{VP} / \mathrm{ml}{ }^{125}$ I-RSOAds-hTERT/PSA virus solutions, 3 -min reaction duration, $\mathrm{pH} 7.5$ and $120 \mu \mathrm{l}$ of PBS.

Measurements of radiochemical purity. After isolation and purification of ${ }^{125} \mathrm{I}$-RSOAds-hTERT/PSA by gel-filtration chromatography, the radiochemical purity of markers, assayed by the paper chromatography, was $>95 \%$. The results of radiochemical purity at difference time points are shown in Fig. 7. After 7 days of preservation in the refrigerator at $4^{\circ} \mathrm{C}$, the radiochemical purity of ${ }^{125}$ I-RSOAds-hTERT/PSA was stable at approximately $93-94 \%$. 


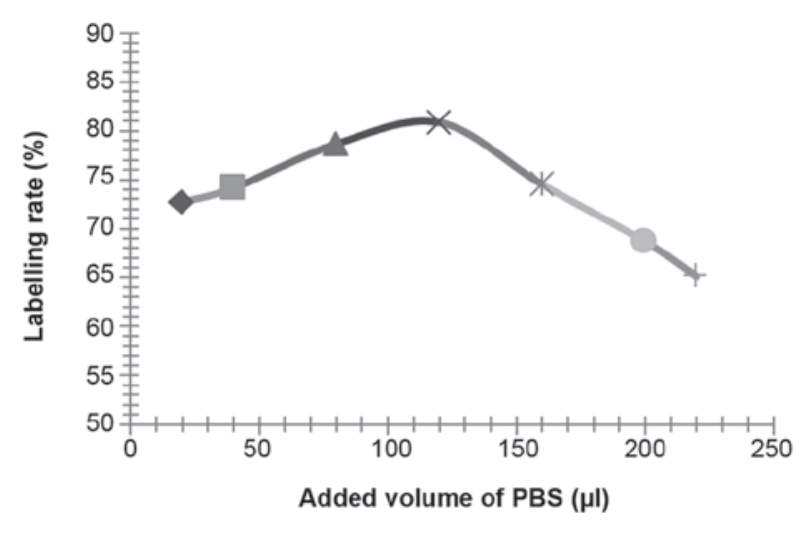

Figure 6. Influence of added volume of PBS on the labelling rate.

\section{Discussion}

As a newly emerging targeted gene therapy of tumors, RSOAds therapy is used to induce the virus to selectively replicate and exert the oncolytic effect inside tumor cells by genetic transformation of the virus. Selective oncolytic effect, one of the common features of all RSOAds, did not affect normal cells (6). Various viruses could be transformed into oncolytic forms, including adenovirus, retrovirus, herpes simplex virus, vaccinia virus and measles virus. The oncolytic adenovirus, transformed from the adenovirus, has been the research hotspot due to a better understanding and various research of the adenovirus, including its genomic structure, function, mobidity and safety (7). Studies have shown that the deletion of E1A region of virus would not impair the virus infection ability and its intracellular selective replication ability. Under the conditions of deletion of E1B-55 kDa, virus could retain its intracellular selective replicative ability in cells with p53 mutation, but its survival time would be affected, thus reducing the oncolytic activity. The expression of E3-11.6 kDa and E4 would enhance the viral cytotoxic effect. Depletions of E3 10.4/14.5/14.7 regions would enhance the sensitivity to cytokine and antitumor immunity (8). Satoh et al found obvious suppression effect of AxdAdB-3, the oncolytic adenovirus dual-regulated by E1A mutation and E1B depletion, on the prostate cancer cells DU145, PC3 and LNCaP, in which the results of animal experiments also indicated its significant anti-prostate cancer effect (9). Cherubini et al also confirmed that oncolytic adenovirus $\operatorname{Ad} \Delta \Delta$ with the depletions of E1A-CR2 and E1B-19K was a very effective targeted-gene carrier in treatment of prostate cancer and pancreatic cancer (10). In our studies, it was confirmed that dual-regulated oncolytic adenovirus could be constructed with the E1A and E1B genes of virus being regulated by hTERT promoter and HIF-1 promoter, which would serve as a carrier in treatment of bladder cancer by targeted super-antigen SEA, and better targeted effect and killing tumor cell effect were indicated in in vitro experiments (5).

${ }^{125} \mathrm{I}$, a kind of synthetic radioactive nuclides, could emit gamma-rays with low energy and a short distance of only $1.7 \mathrm{~mm}$ after transplantation into the body. Low dosage exposure of ${ }^{125}$ I inhibited mitosis of tumor cells for the integrity of DNA of tumor cells in mitotic phase which is impaired by the gamma-ray, thus leading to the death of tumor cells,

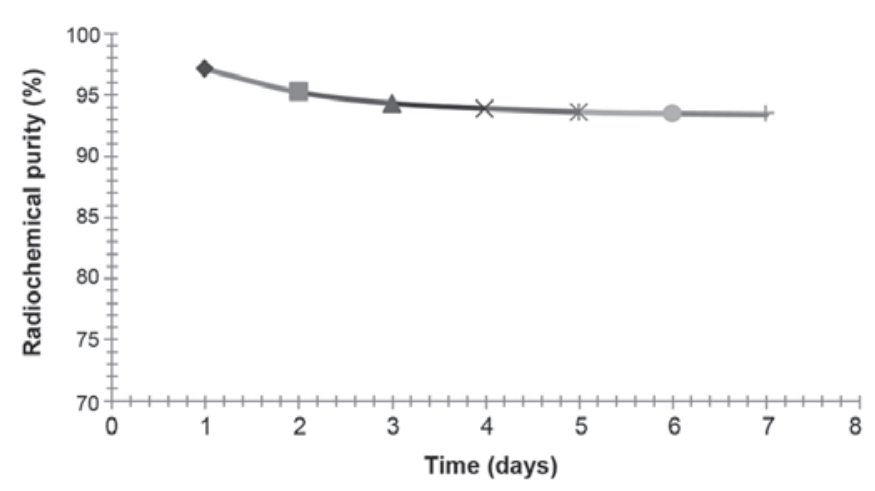

Figure 7. Radiochemical purity at different time points.

instead of continuous mitosis. Moreover, gamma-ray could also re-oxidize the hypoxic cells to increase the sensitivity of tumor cells to gamma-ray (11). Zhu et al found that ${ }^{125} \mathrm{I}$, the radioactive particle, through significantly lowering the rate of positive cells in vascular endothelial growth factor and microvascular density in tumor tissues, could effectively destroy the micro-vessel of tumors, limit the vessel formation of the tumor, reduce the blood supply to tumors, thereby killing the tumor cells and lessening the chance of tumor metastasis (12). Since the short-distance radiotherapy could easily realize a high-dosage exposure to local part of tumor tissues with less influence on surrounding normal tissues but significantly improved clinical efficacy and fewer incidences of complications, ${ }^{125} \mathrm{I}$ is one of the major methods in treatment of prostate cancer in many clinical practices. However, for cases with surrounding fascia or seminal vesicle infiltrated by tumor cells, or metastasis in other major organs or pelvic lymph nodes, or even uncontrollable osseous metastasis, simple application of ${ }^{125}$ I could scarcely achieve radical cure, but should be complemented or combined with other effective treatments (13).

We successfully incorporated the ${ }^{125} \mathrm{I}$ into the ${ }^{125} \mathrm{I}$ RSOAds-hTERT/PSA markers to produce the targeted effect on the prostate cancer cells, which could be expected to kill the tumor cells specifically and increase the clinical efficacy of CRPC. Twigger et al found that not only could reovirus exert the synergistic effect with radiation exposure in in vitro experiments to promote the apoptosis of tumor cells, but also its effect was not affected by the sequence of radiation (14). Animal experiments also confirmed that the control effect of combination of reovirus and radiation exposure was significantly higher than that of simple application of virus or radiation exposure. Chen et al found that combined treatment by prostate-specific adenovirus (CV706) and radiotherapy could exert the synergistic suppression effect on the tumor growth after 7-42 days of combined therapy for the heterotopic model of prostate cancer. Besides, after 6 weeks of combined therapy, the prostate-specific antibody level was significantly lower ( $\sim 1 \%$ of the value in control group), showing more significant effect than simple application of virus ( $\sim 86 \%$ of the control group) or radiotherapy ( $139 \%$ of the control group) (15). Dilley et al (8) confirmed that radiotherapy combined with another kind of prostate-specific adenovirus (CV787) could significantly lessen the average volume of tumor $(\sim 34 \%$ of the volume in control group); complete regression of tumor 
in mice was found to increase to $80 \%$ after at least 8 weeks of therapy. Moreover, an obvious decrease was found in serum level of prostate-specific antibody in subjects who accepted combined therapy compared to those who accepted simple adenovirus or radiotherapy.

At present, various shortages remain in treatment of tumors by simple oncolytic adenovirus or radioactive nuclide, thus we need to take some measures to enhance the antitumor ability of virus and nuclide. Labelling the oncolytic adenovirus with radioactive nuclide to exert the oncolytic and radiation effect, by which the cancer cells would be destroyed, thus improving the efficacy of treatment, could be an effective option to enhance the antitumor effect of oncolytic adenovirus, and contribute to an accurate distribution of radioactive nuclide around the tumor cells, which could lessen the dosage of radioactive nuclide and relieve its influence on the normal tissues. Attempts have already been made to treat tumors with an enhanced synergistic effect produced by oncolytic adenovirus labelled by radioactive nuclide (16).

For prostate cancer tissues, hTERT has been currently reported as the cancer biomarker with the widest spectrum, while the PSA is the widely-accepted molecular marker for prostate cancer. If the dynamic integration between these two markers were realized, they would be the best combined tumor markers for prostate cancer. In recent 5 years, we have been working on the RSOAds targeted gene therapy for tumors of urinary system. According to the pathologic and immunological characteristics, we have successfully constructed the dual-targeted and dual-regulated tumor-specific oncolytic adenovirus carrier by PSA and hTERT to enhance the target effect of the therapeutic gene on the tumor tissues of prostate, which is expected to acquire better efficacy. This technique has applied for the European Patent (European Patent Application no. 10153380.0, February 11, 2010).

The oncolytic adenovirus labelled by ${ }^{125} \mathrm{I}$ and dual-regulated by hTERT-PSA promoter possesses high target effect and the carried ${ }^{125}$ I could be administered by local injection or intravenous injection. After the tumor cells are infected by the virus, the nuclide, with the replication of virus, would be separated from the dual-regulated oncolytic adenovirus and concentrated in the tumor cells, thus realizing an accurate distribution of ${ }^{125} \mathrm{I}$ in the tumor cells and overcoming the deficiency that the nuclide could hardly be applied in the targeted therapy in clinical practices. ${ }^{125} \mathrm{I}$, in addition to the continuous killing effect on the tumor cells, it could also consecutively emit the short-distance ionizing radiation to the oncolytic adenovirus, making the tumor stem cells which are resistant to radiotherapy or could not be killed by oncolytic adenovirus more susceptible, and jointly exerting a better killing effect on the prostate tumor cells. For the dosage of oncolytic adenovirus and radioactive nuclide, it might be possible to reduce the dosage in theory to alleviate or avoid the toxic side effect of radioactive nuclide.

Thus, we infer that the combination of oncolytic adenovirus and radioactive nuclide, in addition to the direct killing effect on tumor cells, could exert the targeted-therapeutic effect on the tumor microenvironment, which could remedy the deficiency when they were applied individually: The targeted infection of tumor cells by oncolytic adenovirus could increase the sensitivity of tumor tissues to the radiotherapy, and the radiation could also enhance the oncolytic ability of oncolytic adenovirus. The synergistic effect of which could effectively inhibit the growth of tumor stem cells which were resistant to the radiotherapy and were difficult to kill by the oncolytic adenovirus to achieve the radical treatment of the tumor. Besides, a series of influences would be exerted on the tumor growth microenvironment by the suppression effect of radiation on the tumor micro-vessels and a certain degree of antitumor immunity induced by oncolytic virus to realize the objective of targeted-therapy for tumor microenvironment.

So far, there are few reports on the application of in vitro construction of ${ }^{125}$ I-RSOAds-hTERT/PSA marker for targeted treatment of CRPC. The results of the present study indicated that the radiochemical purity of ${ }^{125} \mathrm{I}$-RSOAds-hTERT/PSA reached $>95 \%$ and its stability could be maintained at $4{ }^{\circ} \mathrm{C}$ for 7 days, at approximately 93-94\%. These results serve as the experimental basis for later antitumor experiments with markers, and are expected to provide new options for the treatment of prostate cancer.

\section{Acknowledgements}

The present study was supported by the Research and Development of Key Programs of Jiangsu (no. BE2015623), the Nation Science Foundation of China (no. 81272557), the Natural Science Foundation of Jiangsu (no. BK2012647), the Peak Projects for 6 kinds of Genius of Jiangsu (no. WSW-185), the International S\&T Cooperation Program of China (no. 2014DFA31480), the Scientific and Technological Programs of Xuzhou (no. XM13B079), the Jiangsu Province Medical Young Talent (QNRC2016386) and the Medical Innovation Team of Jiangsu Province (CXTD-2016-48).

\section{References}

1. Center MM, Jemal A, Lortet-Tieulent J, Ward E, Ferlay J, Brawley $\mathrm{O}$ and Bray $\mathrm{F}$ : International variation in prostate cancer incidence and mortality rates. Eur Urol 61: 1079-1092, 2012.

2. Siegel R, Naishadham D and Jemal A: Cancer statistics, 2013. CA Cancer J Clin 63: 11-30, 2013.

3. Li J, Djenaba JA, Soman A, Rim SH and Master VA: Recent trends in prostate cancer incidence by age, cancer stage, and grade, the United States, 2001-2007. Prostate Cancer 2012: 691380, 2012.

4. Ottolino-Perry K, Diallo JS, Lichty BD, Bell JC and McCart JA: Intelligent design: Combination therapy with oncolytic viruses. Mol Ther 18: 251-263, 2010.

5. Hu J, Xuan X, Han C, Hao L, Zhang P, Chen M, He H, Fan T and Dong B: Anti-tumor function of double-promoter regulated adenovirus carrying SEA gene, in the treatment of bladder cancer. Cell Biochem Biophys 62: 353-359, 2012.

6. Gil Z, Rein A, Brader P, Li S, Shah JP, Fong Y and Wong RJ: Nerve-sparing therapy with oncolytic herpes virus for cancers with neural invasion. Clin Cancer Res 13: 6479-6485, 2007.

7. Yamamoto M and Curiel DT: Current issues and future directions of oncolytic adenoviruses. Mol Ther 18: 243-250, 2010.

8. Dilley J, Reddy S, Ko D, Nguyen N, Rojas G, Working P and Yu DC: Oncolytic adenovirus CG7870 in combination with radiation demonstrates synergistic enhancements of antitumor efficacy without loss of specificity. Cancer Gene Ther 12: 715-722, 2005.

9. Satoh M, Wang H, Ishidoya $\mathrm{S}$, Abe H, Moriya T, Hamada $\mathrm{H}$ and Arai Y: Oncolytic virotherapy for prostate cancer by E1A, E1B mutant adenovirus. Urology 70: 1243-1248, 2007.

10. Cherubini G, Kallin C, Mozetic A, Hammaren-Busch K, Müller H, Lemoine NR and Halldén G: The oncolytic adenovirus $\operatorname{Ad} \Delta \Delta$ enhances selective cancer cell killing in combination with DNA-damaging drugs in pancreatic cancer models. Gene Ther 18: 1157-1165, 2011. 
11. Bolla M, van Poppel H, Collette L, van Cangh P, Vekemans K, Da Pozzo L, de Reijke TM, Verbaeys A, Bosset JF, van Velthoven R, et al; European Organization for Research and Treatment of Cancer: Postoperative radiotherapy after radical prostatectomy: A randomised controlled trial (EORTC trial 22911). Lancet 366: 572-578, 2005.

12. Zhu X, Palmer MR, Makrigiorgos GM and Kassis AI: Solid-tumor radionuclide therapy dosimetry: New paradigms in view of tumor microenvironment and angiogenesis. Med Phys 37: 2974-2984, 2010.

13. Lagerlöf $\mathrm{JH}$, Kindblom $\mathrm{J}$ and Bernhardt P: 3D modeling of effects of increased oxygenation and activity concentration in tumors treated with radionuclides and antiangiogenic drugs. Med Phys 38: 4888-4893, 2011.

14. Twigger K, Vidal L, White CL, De Bono JS, Bhide S, Coffey M, Thompson B, Vile RG, Heinemann L, Pandha HS, et al: Enhanced in vitro and in vivo cytotoxicity of combined reovirus and radiotherapy. Clin Cancer Res 14: 912-923, 2008
15. Chen Y, DeWeese T, Dilley J, Zhang Y, Li Y, Ramesh N, Lee J, Pennathur-Das R, Radzyminski J, Wypych J, et al: CV706, a prostate cancer-specific adenovirus variant, in combination with radiotherapy produces synergistic antitumor efficacy without increasing toxicity. Cancer Res 61: 5453-5460, 2001.

16. Mi YX, Li YC and Long YH: Imaging of radioiodine-labeled KH901, a tumor-specific oncolytic recombinant adenovirus, in nude mice with human hepatocellular carcinoma. Nucl Med Commun 31: 405-410, 2010. 